\title{
Potencialidades fitoquímicas do melão (Cucumis melo L.) na região Noroeste do Rio Grande do Sul - Brasil.
}

\author{
MULLER, N.G. ${ }^{*}$; FASOLO, D. ${ }^{2}$; PINTO, F.P ${ }^{1 ; B E R T E ̂, ~ R . ~}{ }^{1}$; MULLER, F.C. ${ }^{1}$ \\ ${ }^{1}$ URI, Departamento de Ciências Biológicas, C. postal 464, 98802- 470, Santo Ângelo-Brasil; '2URI Depto. de \\ Ciências da Saúde; *nil@urisan.tche.br
}

\begin{abstract}
RESUMO: O melão (Cucumis melo L.) é uma fruta muito apreciada por suas qualidades e sua produção vem crescendo e ganhando espaço no mercado nacional e internacional. Em regiões como o Noroeste do Rio Grande do Sul, destaca-se como uma nova alternativa de renda para vários agricultores. Neste contexto, o presente trabalho teve como objetivo analisar o potencial fitoquímico de alguns cultivares de melão da região Noroeste do Rio Grande do Sul. A análise fitoquímica utilizando como farmacógeno as folhas, foi realizada para a verificação da presença de metabólitos secundários, tais como: saponinas, cumarinas, cardiotônicos, cianogenéticos, alcalóides, taninos, antraquinonas, flavonoides, e óleos voláteis. Também foi avaliado o teor de suco a partir dos frutos. Dentre os cinco cultivares analisados, Gaúcho, Imperial, Hy Mark, Magelan, e Cantaloupe, o cultivar Gaucho apresentou a maior variedade em metabólitos secundários. Na avaliação do teor de suco a cultivar Magelan se destacou em comparação às demais cultivares testadas.
\end{abstract}

Palavras-chave: Cucumis melo L., análise fitoquímica, teor de suco.

ABSTRACT: Phytochemical potentialities of melon (Cucumis melo L.) in the northwest region of Rio Grande do Sul - Brazil. The melon (Cucumis melo L.) is a fruit highly appreciated for its qualities and its production has been growing and gaining space in the national and in the international market. In regions like the northwest of Rio Grande do Sul - Brazil, it stands out as a new income alternative for farmers. In this context, this study aimed to analyze the phytochemical potential of some melon cultivars in the northwest region of Rio Grande do Sul. The phytochemical analysis, using the leaves as pharmacogen, was performed to verify the presence of secondary metabolites such as saponins, coumarins, cardiac glycosides, cyanogenetic glicosides, alkaloids, tannins, anthraquinones, flavonoids and volatile oils. The juice content from the fruits was also evaluated. Among the five analyzed cultivars, Gaucho, Imperial, Hy Mark, Magelan and Cantaloupe, cultivar Gaucho had the greatest variety of secondary metabolites. In the assessment of the juice content, cultivar Magelan was superior compared to the remaining tested cultivars.

Key words: Cucumis melo L., phytochemical analysis, juice content.

\section{INTRODUÇÃO}

Originário da região central da Ásia e da África, o melão (Cucumis melo L.) pertence à família das Cucurbitáceas. Fruto tropical de alto valor comercial, apreciado por suas características peculiares, tanto no mercado interno, quanto no externo, os melões nobres ("cantaloupes") são os preferidos pelo mercado norte americano devido ao sabor, coloração, valor nutritivo (fonte de vitamina A) e uniformidade (Boas et al., 1998; Silva, 2001).

O melão é uma das olerícolas mais populares do mundo, sendo a China o maior produtor responsável por 35\% da produção mundial.
No que se refere às exportações, o Brasil é o sexto maior do mundo; foi introduzido pelos imigrantes europeus, inicialmente cultivado no Rio Grande do Sul (Grangeiro et al., 2002).

O destaque da produção do melão na Região Nordeste do Brasil, acompanha o desenvolvimento da fruticultura que vem ao longo das últimas duas décadas substituindo as atividades agrícolas tradicionais no sertão, como o cultivo de feijão, algodão e milho. Isto se dá pela produção de frutos proporcionar maiores lucros, ocasionando outras oportunidades de crescimento para os produtores, 
sendo atualmente uma das atividades que mais gera emprego e contribui com o superávit da balança comercial no Brasil (Oliveira et al., 2005).

Atualmente, a produção de frutíferas como o meloeiro, vem crescendo e ganhando espaço em várias regiões. No Noroeste do Rio Grande do Sul, por exemplo, diversos tipos de agricultores familiares encontram dificuldade de viabilizar suas unidades produtivas num prazo mais ou menos curto (Dudermel et al., 1993; Noronha, 2005). Nesse contexto, a fruticultura constitui uma alternativa com elevado potencial de retorno econômico para estas famílias e consequentemente para região, destacando a produção de melão por parte de alguns produtores.

Os frutos produzidos possuem forma, tamanho e coloração variáveis, contendo de 200 a 600 sementes, possuindo casca lisa ou levemente enrugada. O meloeiro é uma planta anual, herbácea, com número de hastes ou ramificações variáveis dependendo da cultivar, e considerado um fruto nãoclimatério; as folhas são alternadas e simples. Sua polinização é feita por insetos, principalmente por abelhas. O sistema radicular é bastante ramificado e pouco profundo. Sendo originário de regiões tropicais, as condições de clima quente e umidade relativa baixa favorecem o melhor desenvolvimento das plantas propiciando a alta produtividade e frutos de excelente qualidade (Grangeiro et al., 2002).

Composto basicamente de água e sendo pouco calórico, o melão é uma fruta muito refrescante, podendo ser indicado para vários tipos de dietas alimentares, como para regimes de emagrecimento e para o restabelecimento de doentes em convalescença (Silva, 2001). Assim, a recomendação da introdução do consumo de frutas na dieta alimentar e na cura de determinadas doenças requer maiores pesquisas acerca de suas potencialidades fitoquímicas, fornecendo importantes parâmetros do material vegetal e auxiliando na descoberta de compostos bioativos em frutíferas.

Os componentes bioativos presentes na biodiversidade podem fornecer produtos de grande importância econômica. Dentre eles destacam-se os fitoterápicos e os fitofármacos originados dos recursos genéticos vegetais (Simões et al., 2007).

Os princípios ativos são compostos químicos naturalmente produzidos pelas plantas para protegê-las contra vírus, fungos e podem ser benéficas quando ingeridas pelos humanos através da alimentação de frutas e verduras, por exemplo, prevenindo determinadas doenças. Neste contexto, o presente trabalho teve por objetivo avaliar o potencial fitoquímico e o teor de suco de diferentes cultivares de melão produzido na região Noroeste do Rio Grande do Sul (Simões et al., 2007).

\section{MATERIAL E MÉTODO}

O período de realização deste trabalho contemplou os meses de janeiro e fevereiro de 2008, na Região Noroeste do Rio Grande do Sul. As diversas cultivares de melão foram coletadas em diferentes municípios da região, selecionados aleatoriamente. As amostras representam dois grupos: Cucumis melo L. var. cantaloupensis Naudin - cultivar do tipo Gaúcho e Cucumis melo L. var. reticulatus - cultivares do tipo Cantaloupe, Imperial, Magelan e Hy Mark, as quais foram coletadas na fase em que o fruto encontrava-se maduro, com a casca amarelada.

Após a coleta, as amostras foram depositadas em sacos de papel Kraft, devidamente etiquetadas e identificadas, e encaminhadas ao Laboratório de Botânica da Universidade Regional Integrada do Alto Uruguai e das Missões (URI), campus de Santo Ângelo, para identificação das espécies. As exsicatas foram preparadas com material in natura recém colhido, ainda antes da formação do fruto, quando em plena fase de floração. O ramo florido foi colocado em uma prensa com papel jornal por sete dias, efetuando-se a substituição por outro papel diariamente. Decorrido o período de desidratação, o material foi fixado com agulha e linha em cartolina, identificado e depositado no HPBR - Herbário Balduíno Rambo da Universidade Regional Integrada do Alto Uruguai e Missões (URI) - Campus Erechim. Neste as exsicatas foram registradas com os seguintes números 11.271, 11.272, 11.273, 11.307 e 11.308.

As folhas e frutos foram retirados em diversos exemplares situados ao longo da área de cultivo, objetivando formar uma amostragem de maior representatividade de cada cultivar (Bôas et al., 2002). As folhas foram submetidas à secagem à sombra em ambiente arejado e protegido de insetos. A análise fitoquímica através de métodos clássicos de caracterização (Tabela 1), utilizando como farmacógeno as folhas, e a avaliação do teor de suco, obtido a partir dos frutos, foram realizadas no Laboratório de Farmacognosia da Universidade Regional Integrada do Alto Uruguai e das Missões (URI) - Campus Santo Ângelo.

Para determinar o teor de suco, os frutos foram medidos, pesados e posteriormente triturados em um liquidificador. Filtrou-se o suco em um tecido duplo de algodão, para obter-se apenas o líquido, livre da presença da polpa. Utilizou-se um fruto maduro e inteiro, selecionado aleatoriamente como amostra. O teor de suco foi obtido pela equação abaixo (Boâs et al., 2002):

$$
\text { Teor de suco }=\frac{\text { Peso de suco }}{\text { Peso de amostra }} \times 100
$$


TABELA 1. Métodos utilizados para a análise fitoquímica.

\begin{tabular}{lll}
\hline Metabólitos Secundários & Metodologia utilizada & Referência \\
\hline Óleos voláteis & Extração em aparelho de Clevenger. & Farm. Bras. IV (1988) \\
Flavonóides & Reações: Shinoda, Hidróxidos Alcalinos e UV. & Simões et al. (2004) \\
Alcalóides & Reagentes: Mayer, Dragendorff e Bouchardat. & Costa (2002) \\
Heterosídeos Cardiotônicos & Reações: Kedde, Baljet, Liebermann-Burchard & Macek et al. (1972) \\
Cumarinas & e Keller-Kiliani. & Costa (2002) \\
Saponinas & Fluorescência no UV & Farm. Bras. IV (1988) \\
Taninos & Determinação do índice de espuma. & Simões et al. (2004) \\
Antraquinonas & Reações: Gelatina 2,5\%, Acetato de Chumbo, & Costa (2000) \\
Cianogenéticos & Sulfato de Quinina e Acetato de Cobre. & Costa (2000)
\end{tabular}

\section{RESULTADOS E DISCUSSÃO}

Dentre as cultivares testadas nenhuma indicou a presença de óleos voláteis. Não existem relatos na literatura quanto à presença ou ausência de óleos voláteis nas folhas do melão para que se pudesse fazer uma análise comparativa. Os óleos voláteis são misturas complexas de substâncias voláteis, geralmente odoríferas e líquidas, também chamados de óleos essências ou essências. Sua principal característica é a volatilidade (Simões et al., 2002). Alguns óleos essenciais são conhecidos por suas propriedades antioxidantes, sendo de grande aplicação na indústria farmacêutica, de cosméticos e na produção de alimentos (Yunes \& Cechinel Filho, 2007).

Em relação à determinação de saponinas, as cultivares Gaúcho e Magelan apresentaram resultados positivos, destacando a maior quantidade destes compostos na cultivar Gaúcho (Tabela 2). As saponinas apresentam propriedades detergentes e emulsificantes, e também são empregadas na indústria farmacêutica por suas propriedades expectorante, diurética e antiinflamatória (Simões et al., 2002).

Amplamente encontrados no reino vegetal, os taninos apresentaram resultado positivo nas reações com acetato de chumbo e acetato de cobre para todas as cultivares avaliadas (Tabela 2). Estes compostos estão relacionados principalmente por sua propriedade adstringente. Além disso, promovem efeitos antidiarréico, antisséptico, antimicrobiano e protegem as plantas contra ataques de herbívoros e microrganismos; são utilizados na tanagem (curtimento) do couro, de onde surgiu a nomenclatura desta classe de metabólitos (Monteiro, 2005).

Em relação aos alcalóides, nenhuma das cultivares analisadas neste estudo apresentou positividade nos testes realizados (Tabela 2), possivelmente, por serem substâncias de distribuição restrita.

As antraquinonas, além de serem consideradas corantes naturais também apresentam propriedades laxativas. Após realizada a reação de Bornträger, pôde-se observar a ausência destas substâncias em todo material amostrado (Tabela 3).

Dentre as cultivares analisadas, a do tipo Cantaloupe apresentou uma positividade mais acentuada em relação à presença de compostos flavonoídicos (Tabela 3) em comparação com as outras, que também demonstraram possuir estes compostos. Os flavonóides são substâncias que apresentam inúmeras propriedades, dentre elas

TABELA 2. Avaliação da presença de saponinas, taninos e alcalóides.

\begin{tabular}{lccccccc}
\hline Cultivar & & \multicolumn{3}{c}{ Saponinas } & \multicolumn{2}{c}{ Alcalóides } \\
\cline { 2 - 7 } & & $\begin{array}{c}\text { Acetato de } \\
\text { chumbo }\end{array}$ & $\begin{array}{c}\text { Sulfato de } \\
\text { Quinina }\end{array}$ & $\begin{array}{c}\text { Gelatina } \\
\text { Acetato de } \\
\text { cobre }\end{array}$ & $\begin{array}{c}\text { Mayer Bouchardat } \\
\text { Dragendorf }\end{array}$ \\
\hline Gaúcho & ++ & + & - & - & + & - & - \\
Imperial- & + & - & - & + & - & - & - \\
Cantaloupe & - & + & - & - & +- & - & - \\
Magelan & + & + & - & - & +- & - \\
Hy Mark & - & + & - & - & + & - & - \\
\hline
\end{tabular}

* (+) Presença do metabólito; (-) Ausência do metabólito; (+-) Presença reduzida do metabólito; (++) Presença acentuada do metabólito. 
podemos citar a anti-inflamatória, antimicrobiana, antifúngica e antioxidante (Longhini, 2007).

Os flavonóides são compostos amplamente distribuídos no reino vegetal, representados por diferentes classes de substâncias, sendo as flavonas um dos mais abundantes, possuindo atividades anticancerígenas, além de atuarem em processos reguladores do metabolismo. Encontramse em abundância em várias espécies de frutas e hortaliças, sendo assim, a sua ingestão pode ser benéfica na proteção contra a incidência de diferentes tipos de câncer (Chitarra, 2005).

O interesse econômico dos flavonóides também é decorrente da sua propriedade corante, pois são considerados os pigmentos naturais mais abundantes no reino vegetal, contribuindo no que se refere à atratividade dos alimentos utilizados na dieta (Simões et al., 2002).

Os melões Gaúcho e Imperial possuem entre os seus compostos fitoquímicos as cumarinas (Tabela 3), substâncias encontradas predominantemente em angiospermas. Possivelmente não foi possível identificar a presença de cumarinas em todas as cultivares, por estas serem pertencentes à família Cucurbitácea, a qual não possui destaque na literatura dentre as famílias que apresentam maior número de relatos de compostos cumarínicos. Estas substâncias, pelo seu odor, são muito utilizadas nas indústrias de domissanitários e cosméticos (Simões et al., 2002).
Os compostos cianogenéticos e os cardiotônicos são encontrados com maior facilidade em plantas que apresentam potencial tóxico, sendo mais difíceis de serem encontrados em frutíferas justamente por sua toxicidade. Os resultados mostraram ausência destes compostos para todas as cultivares (Tabela 4) com apenas uma pequena exceção em relação ao teste de LiebermannBurchard, o qual apresentou positividade reduzida para a cultivar Gaúcho. Entretanto, como a reação de Liebermann-Burchard é característica para detectar núcleos esteroidais e triterpênicos, este resultado possivelmente está relacionado à presença do núcleo das saponinas, detectadas anteriormente para esta cultivar.

O maior teor de suco foi obtido com a cultivar Magelan, seguido da Imperial e Cantaloupe (Figura 1). O melão Gaúcho, com menor representatividade, ainda demonstrou proporcionar maior quantidade de suco que a cultivar Hy Mark, sendo este último mais indicado para o consumo in natura, em pedaços.

Com base na análise fitoquímica, a cultivar que apresentou maior variedade em metabólitos secundários foi o Melão Gaúcho, com presença de saponinas, taninos, flavonóides, cumarinas e cardiotônicos. Neste contexto, deve-se levar em consideração alguns fatores que podem influenciar na composição qualitativa e quantitativa dos metabólitos secundários, como a colheita, o clima e a época da realização do experimento (Oliveira,

TABELA 3. Avaliação da presença de antraquinonas, flavonóides e cumarinas.

\begin{tabular}{lccccc}
\hline Cultivar & Antraquinonas & \multicolumn{3}{c}{ Flavonóides } & Cumarinas \\
\cline { 2 - 5 } & & Shinoda & Hidróxidos Alcalinos & UV & + \\
\hline Gaúcho & - & - & +- & +- & ++ \\
Imperial & - & - & + & +- & - \\
Cantaloupe & - & - & + & + & - \\
Magelan & - & - & +- & +- & + \\
Hy Mark & - & - & & +
\end{tabular}

* (+) Presença do metabólito; (-) Ausência do metabólito; (+-) Presença reduzida do metabólito; (++) Presença acentuada do metabólito.

TABELA 4. Avaliação da presença de cianogenéticos e cardiotônicos.

\begin{tabular}{|c|c|c|c|c|c|}
\hline \multirow[t]{2}{*}{ Cultivar } & \multirow[t]{2}{*}{ Cianogenéticos } & \multicolumn{4}{|c|}{ Cardiotônicos } \\
\hline & & Liebermann-Burchard & Kedde & Baljet & Keller-Kiliani \\
\hline Gaúcho & - & +- & - & - & - \\
\hline Imperial & - & - & - & - & - \\
\hline Cantaloupe & - & - & - & - & - \\
\hline Magelan & - & - & - & - & - \\
\hline Hy Mark & - & - & - & - & - \\
\hline
\end{tabular}

*(-) Ausência do metabólito; (+-) Presença reduzida do metabólito. 


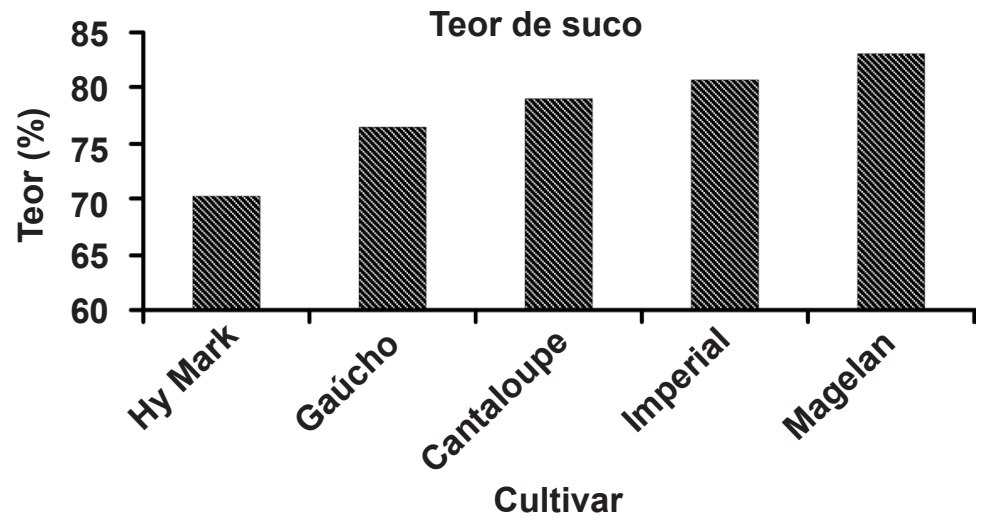

FIGURA 1. Comparação do teor de suco das diferentes cultivares analisadas.

1998). Como exemplo desses fatores podemos citar perdas quantitativas como na redução no peso do alimento, por perda de água ou perda de matéria seca, manuseio inadequado e perdas acidentais. Já nas perdas qualitativas, verificamos os padrões de qualidade, incluindo perdas no sabor, deterioração na textura e aparência (Chitarra, 2005).

Novos experimentos fitoquímicos são necessários em frutíferas, como o melão, por exemplo, pois poucas informações sobre determinadas espécies são encontradas na literatura.

\section{AGRADECIMENTOS}

Ao $\mathrm{CNPq}$ pelo auxílio financeiro, a URI - Campus Santo Ângelo pela disponibilidade dos laboratórios e aos produtores pelo apoio na doação das cultivares.

\section{REFERÊNCIAS}

BOAS, E.V.B.V.; CHITARRA, A.B.; MENEZES, J.B. Modificações dos componentes de parede celular do melão Orange Flesh submetido a tratamento pós-colheita com cálcio. Brazilian Archives of and Tecnology, v.41, n.4, p.467-74, 1998.

BÔAS, R.L.V.; MORAES, M.H.; ZANINI, J.R.; PAVANI, L.C.; CAMARGO, D.A.; DUENHAS, L.H. Teores de nutrientes na folha, qualidade do suco e massa seca de raízes de laranja "valência" em função da irrigação e fertirrigação. Revista Brasileira de Fruticultura, v.24, n.1, p.231-35, 2002.

CHITARRA, M.I.F. Pós-colheita de frutas e hortaliças: fisiologia e manuseio. Lavras: UFLA, 2005. 785p.

COSTA, A.F. Farmacognosia. Lisboa: Fundação Calouste Gulbenkian, 2000. 992p.

COSTA, A.F. Farmacognosia. Lisboa: Fundação Calouste Gulbenkian, 2002. 1117p.

DUDERMEL, T.; BASSO, D.; LIMA, A.P. A Política Agrícola e Diferenciação da Agricultura no Noroeste do RS. ljuí: UNIJUÍ. 1993.

FARMACOPÉIA Brasileira. 4. ed. São Paulo: Atheneu,
1988. 1320p.

GRANJEIRO, L.C.; CECÍLIO FILHO, A.B.; BRAZ, L.T.; GONÇALVES, F.C. Cultivo de melão amarelo. Jaboticabal: Cecílio Filho, A. B., 2002. 30p.

LONGHINI, R.; RAKSA, S.M.; OLIVEIRA, A.C.P.; SVIDZINSKI, T.E.; FRANCO, S.L. Obtenção de extratos de própolis sob diferentes condições e avaliação de sua atividade antifúngica. Revista Brasileira de Farmacognosia. V.17, n.3, p.388-95, 2007.

MACEK, K. Pharmaceutical Applications of Thin-Layer and Paper Chromatography. Amsterdam: Elsevier Publishing Company, 1972. 744p.

MONTEIRO, J.M.; ALBUQUERQUE, U.P.; ARAÚJO, E.L. Taninos: uma abordagem da química a ecologia. Química Nova. V.28, n.5, p.892-96, 2005.

NORONHA, A.D.H. O desenvolvimento da agricultura de Eugênio de Castro: Análise e recomendações de políticas. 2005. Dissertação (Mestrado - Programas de Pós-graduação da CAPES) UNIJUí, ljuí.

OLIVEIRA, A.M.; GURGEI, A.F.; LIMA, L.C.R. Diagnóstico do agronegócio do melão (Cucumis melo L.) produzido em Mossoró/RN: estudo de caso em três empresas produtoras. Holos. v.2, p.27-36, 2005.

OLIVEIRA, F. Farmacognosia. São Paulo: Editora Atheneu, 1998. 412p.

SILVA, S.P. Frutas no Brasil. São Paulo: Nobel, 2001. 230p.

SIMÕES, C.M.O.; SCHENKEL, E.P.; GOSMANN, G.; MELLO, J.C.P.; MENTZ, L.A.; PETROVICK, P.R. Farmacognosia: da planta ao medicamento. 4.ed. Porto Alegre/Florianópolis: UFRGS Editora/ Editora da UFSC, 2002. 833p.

SIMÕES, C.M.O.; SCHENKEL, E.P.; GOSMANN, G.; MELLO, J.C.P.; MENTZ, L.A.; PETROVICK, P.R. Farmacognosia: da planta ao medicamento. $5^{\mathrm{a}}$. ed. Porto Alegre/ Florianópolis: UFRGS Editora/ Editora da UFSC, 2004.

SIMÕES, C.M.O.; SCHENKEL, E.P.; GOSMANN, G.; MELLO, J.C.P.; MENTZ, L.A.; PETROVICK, P.R. Farmacognosia: da planta ao medicamento. $6^{\mathrm{a}}$. ed. Porto Alegre/ Florianópolis: UFRGS Editora/ Editora da UFSC, 2007. 1104p.

YUNES, R.A.; CECHINEL, F.V. Química de produtos naturais, novos fármacos e a moderna farmacognosia. Itajaí: UNIVALI, 2007. 303p. 